\title{
Detection and Management of Pregnancy in Rudimentary Horn of Uterus
}

\author{
Rizwana Parveen
}

\begin{abstract}
Pregnancy in rudimentary horn is extremely rare and usually terminates in rupture during first and second trimester of pregnancy. Diagnosis of rudimentary horn pregnancy and its rupture is difficult and can be missed in routine ultrasound scan; and in majority of cases, it is detected after rupture. It requires high index of suspicion. We present a case report of a woman who was misdiagnosed as having missed miscarriage; and termination was attempted, which ended up in failure and rupture of rudimentary horn was the consequence followed by laparotomy. With advances in prenatal ultrasound in recent decades, it is prudent to detect such a life-threatening condition earlier resulting in a lower incidence of maternal morbidity and mortality.
\end{abstract}

Key Words: Rudimentary horn, Mullarian duct malformation, Unicornuate uterus, Rupture.

\section{INTRODUCTION}

Unicornuate uterus with rudimentary horn is a mullarian duct malformation that results from incomplete development of one of the mullarian ducts and incomplete fusion with the contralateral side. ${ }^{1}$ It is type 2 mullarian duct malformation according to American Fertility Society (AFS) classification. Incidence of mullarian duct malformation is $4.3 \%$ in general population and that of unicornuate uterus is $0.4 \%$. Pregnancy in rudimentary horn is very rare and occurs in $1 / 76,000$ to $1 / 150,000$ pregnancies, but is a potentially lethal condition and associated with high risk of maternal mortality. ${ }^{2}$ Diagnosis is difficult as there is no definitive criteria and usually it is misdiagnosed as ectopic pregnancy or missed miscarriage. Therefore, proper and timely detection and management of this potentially life-threatening condition can drastically reduce maternal mortality.

This case report is presented to emphasise the crucial importance of earlier detection and management for the prevention of life-threatening complications.

\section{CASE REPORT}

A 34-year gravida 5 and para 3+1 with all normal vaginal births and one first trimester miscarriage, presented for the first time to clinic at 15 weeks of pregnancy for antenatal checkup. Her past medical and surgical history was not significant except one evacuation for incomplete

Department of Obstetrics and Gynaecology, Jeddah National Hospital, Jeddah, Saudi Arabia

Correspondence: Dr. Rizwana Parveen, Department of

Obstetrics and Gynaecology, Jeddah National Hospital,

Al Makaronah Street, Jeddah 21492, Saudi Arabia

E-mail:aargrm@yahoo.com

Received: October 11, 2018; Accepted: February 19, 2019 miscarriage 5 years ago. Her previous pregnancies were normal and uneventful. She had normal menstrual periods with no history of dysmenorrhea. All routine investigations were within normal range and ultrasound revealed single viable fetus corresponding to 15 weeks gestation intrauterine pregnancy. She had second scan after 2 weeks when she complained of mild vaginal bleeding, and it showed single viable fetus corresponding to 17 weeks gestation. She was reassured and counselled to follow in the clinic. After 3 weeks, she again had episode of vaginal bleeding and scan revealed intrauterine pregnancy of 17 weeks gestation with no cardiac activity. She was diagnosed as missed abortion and decided for termination of pregnancy. On admission, her vitals were normal with blood pressure of $120 / 70 \mathrm{mmHg}$, pulse 80 beats per minute and she was afebrile. Her baseline blood investigations were normal with haemoglobin of $12.2 \mathrm{~g} / \mathrm{dl}$ and normal platelet count and normal coagulations profile. She was induced with 200 ug misoprostol every 6 hourly of total 5 doses. Patient experienced uterine irritability with minimal vaginal spoting, but no cervical change. Intrauterine Foley's catheter was introduced with prostine gel $1 \mathrm{mg}$. She started dizziness and palpitation with hypotention but on pervaginal examination, no difference in findings were noticed. Provisional diagnosis was made of ectopic pregnancy and ruptured uterus, thus decision was made for laparotomy after arrangement of blood. During surgery, 1.5 litre blood was found in the peritonel cavity. Bicornuate uterus was found with pregnancy in rudimentary horn which was ruptured at fundus. Fetus was found in peritoneal cavity with placenta still in rudimentary horn that was resected and uterus repaired. Rudimentary horn was non-communicating. The main uterine cavity was connected to the cervical canal. Postoperative course was uneventful and she was discharged home on third day. 


\section{DISCUSSION}

Pregnancy in rudimentary horn is a rare obstetric entity but associated with grave risk of maternal morbidity and mortality. Unicornuate uterus with rudimentary horn occurs due to arrested development of mullarian duct. This rudimentary horn is sub-classified into communicating and non-communicating with uterine cavity and horn with no cavity. Around $83 \%$ cases are noncommunicating with main uterine cavity. ${ }^{3}$ The attachment of rudimentary horn to the main uterus varies from fibro-muscular band to an extensive fusion between 2 horns, where there is no separation between them. Approximately $38 \%$ of patients have co-existing renal abnormalities like uni-lateral renal agenesis, always ipsilateral with rudimentary horn. ${ }^{4}$ Conception in rudimentary horn arises either through communication with uterine cavity (communicating) or by transperitoneal migration of fertilised ovum from contralateral side (non-communicating). 5 Diagnosis is suspected from clinical history and examination, which is confirmed by ultrasound and MRI. Uni-cornuate uterus is associated with varying degree of chronic pelvic pain and dysmenorrhea, first trimester miscarriage (24\%), second trimester miscarriage $(9 \%)$, ectopic pregnancy $(27 \%)$, preterm labour $(20 \%)$, intrauterine growth retardation (IUGR), intrauterine fetal demise $5 \%$, placenta accreta and malpresentation. 6,7 Women may present with vague abdominal pain to acute abdomen with haemorrhagic shock or asymptomatically. On clinical examination, uterus is found deviated with palpable adnexal mass. This can be a clue to diagnose rudimentary horn pregnancy, which can be confirmed by ultrasound and MRI. ${ }^{8}$ The sensitivity of ultrasound is only $26-30 \%$, which decreases as pregnancy progresses as the enlarging horn with a thinned myometrium can obscure the adjacent anatomical structures. Some authors have suggested ultrasound criteria described as: (1) pseudo pattern of an asymptomatic bicornuate uterus, (2) absent visual continuation between cervical canal and lumen of pregnancy horn, (3) presence of myometrial tissue surrounding the gestational sac, and (4) hyper vascularisation typical of placenta accreta with colour doppler/power doppler sonography. The ultrasound diagnosis can be confirmed by MRI. Placement of Foley's catheter into uterine cavity prior to performing a transabdominal ultrasound to exclude intrauterine pregnancy is useful but not a preferred method. Immediate surgery should be performed whenever diagnosis is made. Laparotomy is done to prevent rupture and recurrent rudimentary horn pregnancy. Conservative treatment is also tried in selective cases of earlier gestation, like systemic methotrexate, followed by laparoscopy or direct laparoscopic resection, if pregnancy is small. The usual outcome of rudimentary horn pregnancy is rupture in second trimester in $90 \%$ cases with fetal demise. Rupture is due to underdevelopment of myometrium as in this case. If pregnancy progresses beyond 20 weeks, it can lead to IUGR, malformed fetus, oligohydromnios pathological placentation resulting in placenta accreta. Most of the cases remain undiagnosed until rupture or misdiagnosed and usually induction with misoprostol is started which is unsuccessful and can lead to rupture. There should be high index of suspicion to detect the condition in pre-rupture state. 9 Non-responders to induced abortion should be investigated promptly with repeat ultrasound or MRI. Reports of rupture varying from 5-37 weeks are described depending on horn musculature, variable thickness and distensibility of myometrium. ${ }^{10}$ Bleeding from rupture can be drastic and lifethreatning and is controlled using torniquete at uterine isthmus to compress contralateral ovarian and uterine vessels.

In conclusion, conception in rudimentary horn, although rare, can lead to severe obstetrical complications. Diagnosis is challenging and most of the time it is misdiagnosed and managed accordingly until lifethreatening rupture of rudimentary horn occurs. In this case, it was misdiagnosed as missed abortion and induction was tried with misoprostol that was unsuccessful. There should be high index of suspicion if there is no response to misoprostol. Ultrasound should be repeated along with confirmation with MRI. Immediate pre-rupture laparotomy is of crucial importance to prevent morbidity and mortality.

\section{REFERENCES}

1. Khati NJ, Frazier AA, Brindle KA. The unicornuate uterus and its variants: Clinical presentation, imaging findings, and associated complications. J Ultrasound Med 2012; 31: 319-31.

2. Moawad GN, Abi Khalil ED. A case of recurrent rudimentary horn ectopic pregnancies managed by methotrexate therapy and laproscopic excision of the rudimentary horn. Case Rep Obstet Gynecol 2016; 2016:5747524.

3. Kanagal DV, Hanumanalu LC. Ruptured rudimentary horn pregnancy at 25 weeks with previous vaginal delivery: A case report. Case Obst Gyna 2012; 985076.

4. Ambusaidi $Q$, Jha $C$. Pregnancy in the rudimentary uterine horn, a case report of an unusual presentation. Sultan Qaboos Univ Med J 2014; 14:e134-8.

5. Lai YJ, Lin $\mathrm{CH}$, Hou WC, Hwang KS, Yu MH, Su HY. Pregnancy in non-communicating rudimentary horn of a unicornuate uterus: Prerupture diagnosis and management. Taiwan J Obstet Gynecol 2016; 55:604-6.

6. Mei SS, Pariyar J, Yan ZY, Khatri KS, Bishnu P, Jian H. A case report of pregnancy in rudimentary horn. Gynecol Obstet case report 2016, 2:3.

7. Caserta D, Mallozzi M, Meldolesi C, Bianchi P, Moscarini M. 
Pregnancy in unicornuate uterus: a case report. J Med Case Rep 2014; 8:130.

8. Acién P, Acién MI. The history of female genital tract malformation classifications and proposal of an updated system. Hum Reprod Update 2011; 17:693-705.
9. Halima TA, Javaid K. Pregnancy in rudimentary horn of uterus: A case report. Res J Obs Gyna 2011; 4:37-41.

10. Hassan $\mathrm{CH}$, Karim AK, Ismail NA, Omar MH. Case report of ruptured non-communicating right rudimentary horn pregnancy: An acute emergency. Acta Medica (Hradec Kralove) 2011; 54:125-6.

…ํ..... 